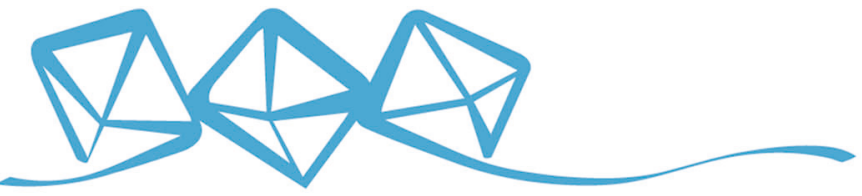 COMMUNICATIONS MATERIALS
}

ARTICLE

https://doi.org/10.1038/s43246-020-0039-9

OPEN

\section{Small bandgap in atomically precise 17-atom-wide armchair-edged graphene nanoribbons}

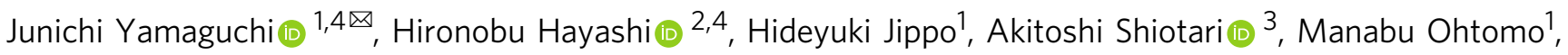
Mitsuhiro Sakakura ${ }^{2}$, Nao Hieda $^{2}$, Naoki Aratani (i) ${ }^{2}$, Mari Ohfuchi ${ }^{1}$, Yoshiaki Sugimoto ${ }^{3}$, Hiroko Yamada (i) ${ }^{2 凶} \&$ Shintaro Sato (1) ${ }^{1}$

Bottom-up synthesis of graphene nanoribbons (GNRs) may open new possibilities in future electronic devices owing to their tunable electronic structure, which depends strongly on their well-defined width and edge geometry. For instance, armchair-edged GNRs (AGNRs) exhibit width-dependent bandgaps. However, the bandgaps of AGNRs synthesized experimentally so far are relatively large, well above $1 \mathrm{eV}$. Such a large bandgap may deteriorate device performance due to large Schottky barriers and carrier effective masses. Here, we describe the bottom-up synthesis of AGNRs with smaller bandgaps, using dibromobenzenebased precursors. Two types of AGNRs with different widths, namely 17 and 13 carbon atoms, were synthesized on $\mathrm{Au}(111)$, and their atomic and electronic structures were investigated by scanning probe microscopy and spectroscopy. We reveal that 17-AGNRs have the smallest bandgap, as well as the smallest electron/hole effective mass, among bottom-up AGNRs reported so far. The successful synthesis of 17-AGNRs is a significant step toward the development of GNR-based electronic devices.

\footnotetext{
${ }^{1}$ Fujitsu Laboratories Ltd. and Fujitsu Limited, 10-1 Morinosato-Wakamiya, Atsugi, Kanagawa 243-0197, Japan. ${ }^{2}$ Division of Materials Science, Nara Institute of Science and Technology, 8916-5 Takayama-cho, Ikoma, Nara 630-0192, Japan. ${ }^{3}$ Department of Advanced Materials Science, The University of Tokyo, Kashiwa, Chiba 277-8561, Japan. ${ }^{4}$ These authors contributed equally: Junichi Yamaguchi, Hironobu Hayashi. ${ }^{凶}$ email: yamaguchi.j@fujitsu.com;

hyamada@ms.naist.ac.jp
} 
Q uasi-one-dimensional strips of graphene with nanoscaled width, so-called graphene nanoribbons (GNRs), exhibit unique electronic and magnetic properties that do not appear in two-dimensional graphene ${ }^{1-4}$. These properties can emerge from the structural boundary conditions imposed by the atomically precise width and edge structure of GNRs. Two representative types of GNRs are armchair-edged GNRs (AGNRs) and zigzag-edged GNRs (ZGNRs). AGNRs show sizable gapped electronic states established by the quantum confinement and edge effects ${ }^{5-8}$. In graphene-based electronics, AGNRs with finite bandgaps at room temperature have attracted much attention for their potential reliability as semiconducting channels for field-effect transistors (FETs). Numerous top-down approaches have been conducted to fabricate GNRs ${ }^{9-11}$. Nevertheless, such GNRs typically had uncontrollable edge geometries with plenty of defects, exhibiting poor transport properties as transistor channels.

For fabricating atomically precise GNRs, an advanced bottomup synthesis technique has recently been proposed and demonstrated, which uses on-surface-assisted polymerization and subsequent cyclodehydrogenation of precursor monomers on metal substrates $^{12}$. This technique has yielded atomically precise AGNRs $^{12-14}$ and ZGNRs ${ }^{15}$. In particular, the bottom-up synthesis of $N$-AGNRs (where $N$ is the width measured by the number of rows of carbon atoms across the AGNRs) has been widely conducted because the bandgap of AGNRs can be tuned by changing their width. Following the pioneering work on 7-AGNR on $\mathrm{Au}(111)^{12}$, various $N$-AGNRs have been reported, such as 3$\mathrm{AGNR}^{16,17}, 5-\mathrm{AGNR}^{18}, 9-\mathrm{AGNR}^{19}$, and 13-AGNR ${ }^{20}$, as well as the fused AGNRs prepared by cyclodehydrogenation along the edge direction (e.g., 14-AGNR, 18-AGNR, and 21-AGNRs) ${ }^{21,22}$.

As theoretically predicted, $N$-AGNRs can be categorized into three subfamilies with $N=3 p, 3 p+1$ and $3 p+2$ ( $p$ is a natural number), in which each electronic structure varies depending on the structural boundary conditions ${ }^{6,7}$. For evaluating the quasiparticle bandgaps of AGNRs accurately, first-principles calculations using many-body perturbation theory ( $G W$ approximation ${ }^{23}$, where $G$ is Green's function and $W$ is the screened Coulomb interaction) have been carried out beyond the framework of the density functional theory $(\mathrm{DFT})^{7}$. According to these calculations, the gap size $\Delta$ decreases with increasing $N$ (i.e., the width) within each subfamily and follows a relation among subfamilies with the same $p$, namely $\Delta^{3 p+1}>\Delta^{3 p}>\Delta^{3 p+2}$. For instance, for the $G W$ calculations for freestanding $N$-AGNRs ${ }^{7,24}$, the quasiparticle gap $\Delta_{G W}$ is predicted to be 3.80 and $2.25 \mathrm{eV}$ for $N=7$ and $13(3 p+1)$, respectively, 2.16 $\mathrm{eV}$ for $N=9(3 p)$ and $1.75 \mathrm{eV}$ for $N=5(3 p+2)$. Previous studies on FETs using bottom-up AGNRs with widths of $N=7,9$ and 13 presented their electrical transport characteristics ${ }^{25,26}$. However, in such GNR-based FETs, the intrinsic transport properties of AGNRs were obscured by a large Schottky barrier resistance between the AGNRs and metal contacts, as a result of the large bandgaps in the above AGNRs, substantially exceeding $1 \mathrm{eV}$. These transport characteristics can be improved through the use of wider AGNRs, as they are expected to have lower Schottky barriers and smaller effective masses arising from their small bandgap features. In fact, in carbon nanotubes (CNTs) having bandgaps smaller than $1 \mathrm{eV}$, much better transport characteristics have been reported ${ }^{27,28}$. Hence, AGNRs belonging to the $3 p+2$ subfamily, which exhibit the smallest bandgaps among the three subfamilies, have considerable potential to be exploited in GNR-based FETs. GNR-based FETs can have an advantage over CNT-based FETs because the structure, and therefore the properties of the GNRs, can be precisely controlled using bottom-up synthesis, in contrast to CNTs.

Here, we discuss the bottom-up synthesis of well-structured 17 -AGNRs $(3 p+2)$, as well as 13 -AGNRs $(3 p+1)$, on $\mathrm{Au}(111)$ in ultra-high vacuum by using two types of dibromobenzene- based precursor monomers. To systematically control the width of these AGNRs in the monomers, either two anthracene or naphthalene units were attached at the ortho-positions of $p$ dibromobenzene. Although 13-AGNRs synthesized with a different precursor monomer have already been reported ${ }^{20}$, we show that our 13-AGNRs are consistent in their properties with the published ones. Characterization of the atomic and electronic structures of the 17-AGNR and 13-AGNR on $\mathrm{Au}(111)$ is carried out by combining in situ scanning tunneling microscopy and spectroscopy (STM/STS) and ex situ non-contact atomic force microscopy (nc-AFM). We find that the experimentally obtained electronic structures in both AGNRs are consistent with the accordingly corrected quasiparticle states predicted from the $G W$ calculations. We reveal that the 17-AGNRs have a bandgap of $0.19 \mathrm{eV}$ on $\mathrm{Au}(111)$, which is consistent with a theoretically obtained bandgap of $0.63 \mathrm{eV}$ for a freestanding $17-\mathrm{AGNR}^{24}$. As far as we know, this is the first demonstration of the synthesis of GNRs having a bandgap smaller than $1 \mathrm{eV}$ in a controlled manner.

\section{Results and discussion}

Synthesis of 17- and 13-AGNRs. To control the width of bottom-up AGNRs, we developed two dibromobenzene-based precursor monomers, as shown in Fig. 1. The monomers 1,2-bis(2-anthracenyl)-3,6-dibromobenzene (BADBB) for the 17AGNRs (Fig. 1a) and 1,2-bis-(2-naphthalenyl)-3,6-dibromobenzene (BNDBB) for the 13-AGNRs (Fig. 1b) were successfully obtained through multi-step organic synthesis (see Supplementary Note 1). Their characterizations were carried out by nuclear magnetic resonance (Supplementary Figs. 1, 2 and 3, 4 for BADBB and BNDBB), mass spectrometry (Supplementary Figs. 5, 6 and 7, 8 for BADBB and BNDBB) and X-ray crystallographic (Supplementary Fig. 9, dataset 1 and Fig. 10, dataset 2 for BADBB and BNDBB) analyses. Annealing a $\mathrm{Au}(111)$ surface up to $250^{\circ} \mathrm{C}$ induced the detachment of bromine atoms from BADBB and BNDBB and formed extended one-dimensional 17-polymer and 13-polymer by aryl-aryl coupling. The covalent bonds between the monomers can form only when they are rotated $180^{\circ}$ to each other, as in the synthesis of 9-AGNRs based on a precursor monomer $3^{\prime}, 6^{\prime}$-dibromo-1,1 $1^{\prime}: 2^{\prime} 1^{\prime \prime}$-terphenyl (DBTP) ${ }^{19,29}$. Upon further annealing to $400^{\circ} \mathrm{C}$, thermally induced cyclodehydrogenation in the polymers led to the formation of the 17AGNR and 13-AGNRs. The BADBB or BNDBB monomers were individually deposited onto a clean $\mathrm{Au}(111)$ surface maintained at room temperature by thermal sublimation under ultra-high vacuum conditions. After room-temperature deposition, it was confirmed using STM that both BADBB and BNDBB monomers tend to form self-assembled islands on $\mathrm{Au}(111)$ (Supplementary Fig. 11a, b). Their apparent heights determined from STM are $0.24 \mathrm{~nm}$ for BADBB and $0.22 \mathrm{~nm}$ for BNDBB. To lengthen the ribbon and decrease the defects in AGNRs, the samples were annealed stepwise from room temperature to $400{ }^{\circ} \mathrm{C}$ in increments of $50^{\circ} \mathrm{C}$, instead of the conventional two-step annealing at 200 and $400^{\circ} \mathrm{C}$ used in previous bottom-up GNRs ${ }^{12,19,20}$.

We successfully observed the formation of the polymers and AGNRs using STM. Figure $2 \mathrm{a}$ shows a large-scale STM topographic image of 17-polymers on $\mathrm{Au}(111)$ after annealing at $250^{\circ} \mathrm{C}$. The 17 -polymers also assemble into extended islands in a manner similar to the BADBB monomers after roomtemperature deposition. The apparent height of the 17-polymers, however, slightly increases to $0.34 \mathrm{~nm}$ compared with $0.24 \mathrm{~nm}$ for the BADBB monomers. This increase is attributed to the sterically induced out-of-plane rotation of the anthracene units after the polymerization. A small-scale STM image of the 17-polymers is presented in Fig. 2b, together with a structural model obtained 
a

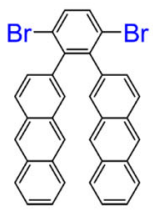

BADBB

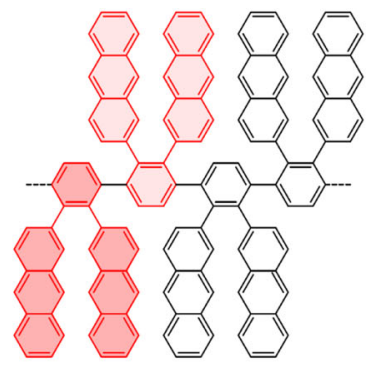

17-polymer $250^{\circ} \mathrm{C}$

$\mathrm{Au}(111)$

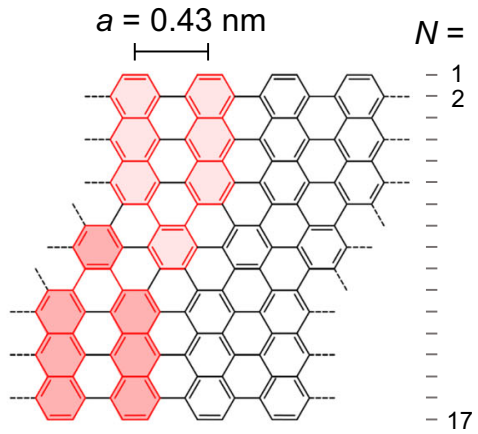

17-AGNR

$400^{\circ} \mathrm{C}$

$\mathrm{Au}(111)$
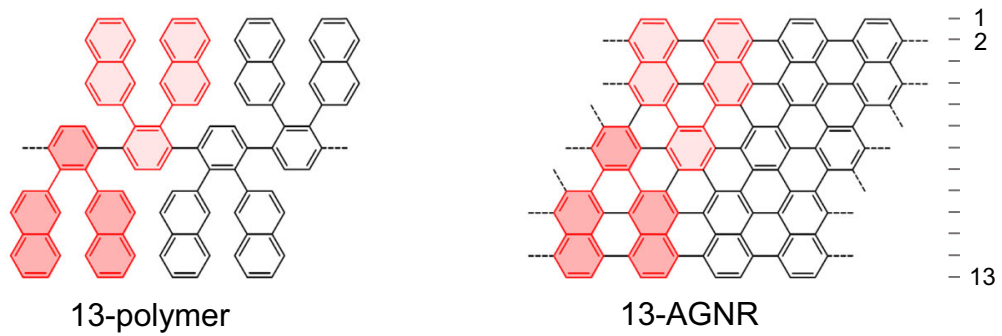

Fig. 1 Bottom-up synthesis of 17- and 13-AGNRs. a, b Schematic drawing of on-surface chemical reactions for a 17- and $\mathbf{b}$ 13-AGNRs on Au(111). 17- and 13-polymers are formed via dehalogenation and aryl-aryl coupling of BADBB and BNDBB monomers up to $250^{\circ} \mathrm{C}$, then cyclodehydrogenation of $17-$ and 13 -polymers at $400^{\circ} \mathrm{C}$ leads to formation of 17- and 13-AGNRs. The relevant lattice parameter of AGNRs is described as $a=0.43 \mathrm{~nm}$ in $\mathbf{a}$.

from DFT calculations for a freestanding 17-polymer. As seen from the STM image, the protrusions, derived from the sterically hindered anthracene units, align with a periodicity of $0.86 \pm 0.05$ $\mathrm{nm}$ along the polymer axis. From the calculations, the predicted periodicity of a repeat unit is $0.90 \mathrm{~nm}$, which is in good agreement with the experimental value. In addition, the simulated STM image of the 17-polymer (Fig. 2c) reproduces well the experimentally observed periodic protrusions.

In the case of the 13-polymers, the large-scale STM image (Fig. 2d) indicates that they exhibit a similar island formation to that of the 17-polymers. The apparent height increases from 0.22 $\mathrm{nm}$ for the BNDBB monomers to $0.32 \mathrm{~nm}$ for the 13-polymers. The small-scale STM image and DFT-simulated image for the 13polymers are presented in Fig. 2e, f. The periodicity of the protrusions arising from the sterically hindered naphthalene units is estimated to be $0.95 \pm 0.08 \mathrm{~nm}$ along the polymer axis. This value agrees with a periodicity of $0.90 \mathrm{~nm}$ predicted from the calculations, and the experimental STM image closely matches the simulated image.

Further annealing to $400{ }^{\circ} \mathrm{C}$ results in a complete planarization of the protruded polymers and leads to fully conjugated AGNRs, which is observed as a reduced apparent height of $0.18 \mathrm{~nm}$ in both 17-AGNRs and 13-AGNRs (Fig. 3a, d). Each width is estimated to be 2.5 and $1.9 \mathrm{~nm}$ with an accuracy of $\pm 0.1 \mathrm{~nm}$ in the 17-AGNRs and 13-AGNRs, respectively. The dimensional feature of these 13-AGNRs is consistent with those synthesized using a different precursor monomer ${ }^{20}$. We notice that the 17-AGNRs and 13-AGNRs have some defects along their edges due to the lack of the anthracene and naphthalene units and are somewhat more defective compared with 9-AGNRs synthesized using DBTP ${ }^{19,29}$. BADBB (for the 17-AGNR) and BNDBB (for the 13-AGNR) have the same molecular backbone ( $p$-dibromobenzene) as DBTP. While two phenyl units were attached to the p-dibromobenzene in DBTP, two anthracene and naphthalene units are attached to the $p$-dibromobenzene in BADBB and $\mathrm{BNDBB}$, respectively. As the six-membered rings of the acene unit increase, the steric hindrance between the two acene units becomes large, resulting in the breaking of the attached acene units during cyclodehydrogenation in the formation of AGNRs. Hence, the defects may tend to be more easily induced in the 17AGNRs and 13-AGNRs than the 9-AGNRs. The high-resolution STM images of the 17-AGNRs and 13-AGNRs without the defects are shown in Fig. 3b, e, together with their structural models of freestanding 17-AGNRs and 13-AGNRs calculated by DFT. The observed molecular structures exhibit an edge periodicity of $0.42 \pm 0.03 \mathrm{~nm}$ along the ribbon axis in both AGNRs. The experimental periodicity is in good agreement with the simulated value $(0.43 \mathrm{~nm})$. The observed ring-like shapes within the AGNRs also agree with the simulated STM images (Fig. 3c for 17-AGNR and Fig. 3f for 13-AGNR).

To check further structural details on the 17- and 13-AGNRs, we carried out ex-situ nc-AFM imaging using CO-functionalized tips. Samples were transferred through the air into the AFM measuring chamber and subsequently annealed at about $400{ }^{\circ} \mathrm{C}$ under ultra-high vacuum conditions to remove contaminations on the surface adsorbed during air exposure. Figure 4a shows the frequency shift image of the 17-AGNR. For better visibility, the corresponding Laplace-filtered image is depicted in Fig. 4b. The Laplace-filtered image directly reveals the width consisting of 17 carbon atoms corresponding to the expected 17-AGNR structure. For the 13-AGNR, the frequency shift image and Laplace-filtered one are presented in Fig. 4c, d. The width consisting of 13 carbon atoms is also confirmed in Fig. 4d. Note that despite ex-situ sample preparation, we were able to obtain the bond-resolved AFM images of bottom-up GNRs. Our results indicate that ncAFM has versatile usability for probing atomic structures, even 
a

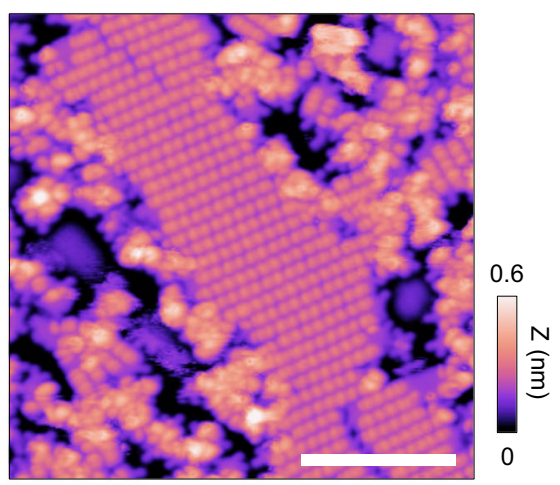

b

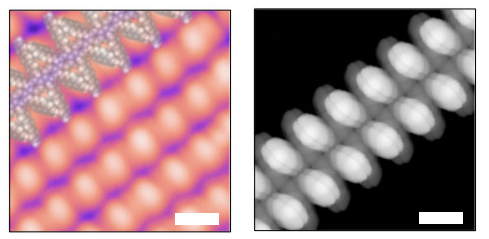

d

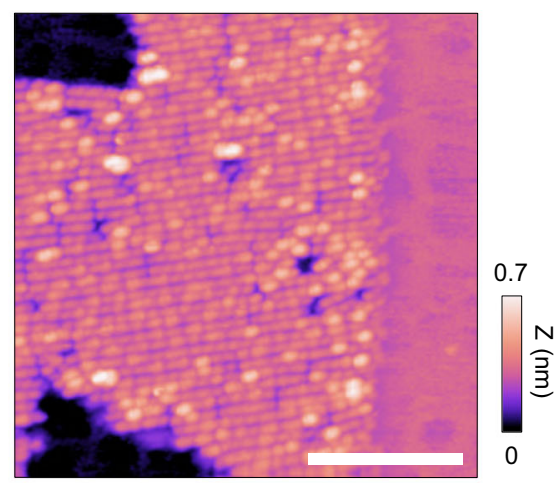

e

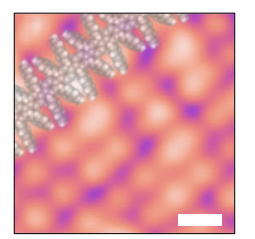

f

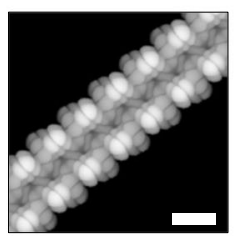

Fig. 2 STM topographies and DFT simulations for 17- and 13-polymers. a Large-scale STM image (scanning paremeters: sample bias $V_{s}=1.1 \mathrm{~V}$, tunneling current $\left.I_{\mathrm{t}}=10 \mathrm{pA}\right)$ of 17-polymers on $\mathrm{Au}(111)$ and $\mathbf{b}$ small-scale image $\left(V_{\mathrm{s}}=1.1 \mathrm{~V}, I_{\mathrm{t}}=10 \mathrm{pA}\right)$. Structural model of single 17-polymer is superimposed in b. c DFT simulated image (energy window $U=1.1 \mathrm{eV}$ ) of the 17-polymer. $\mathbf{d}$ Large-scale STM image $\left(V_{s}=-1.2 \mathrm{~V}, I_{t}=70 \mathrm{pA}\right)$ of 13-polymers on Au(111) and e small-scale image $\left(V_{s}=1.0 \mathrm{~V}, I_{t}=10 \mathrm{pA}\right)$ together with structural model. $\mathbf{f}$ DFT simulated image $(U=1.0 \mathrm{eV})$ of the 13-polymer. All STM images were taken at $77 \mathrm{~K}$. Scale bar, $10 \mathrm{~nm}$ in $\mathbf{a}, \mathbf{d}, 1 \mathrm{~nm}$ in $\mathbf{b}, \mathbf{c}, \mathbf{e}, \mathbf{f}$.

a

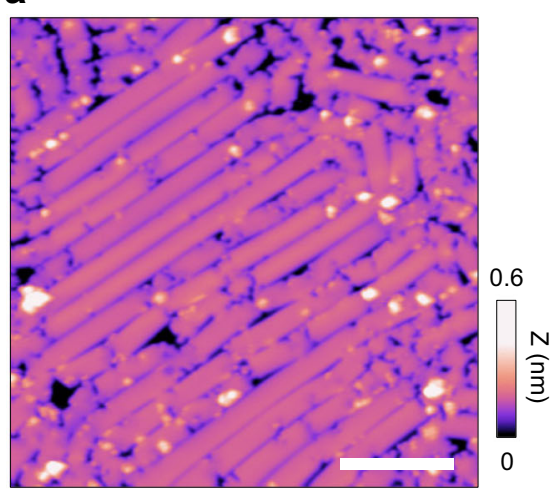

b

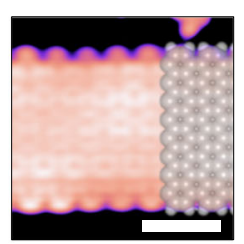

C

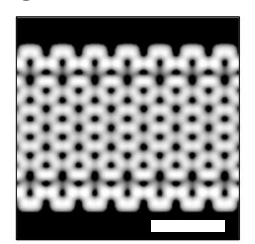

d

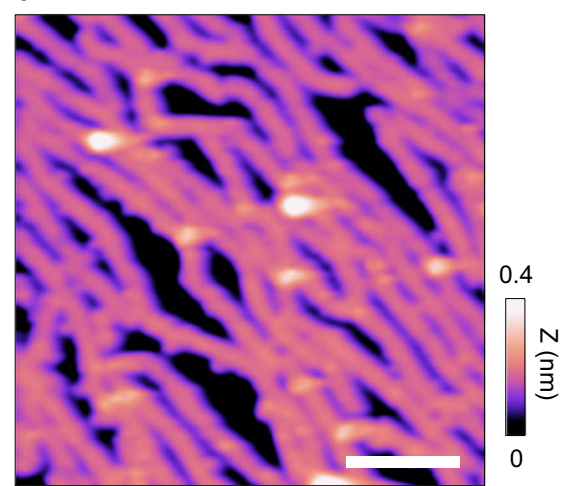

e

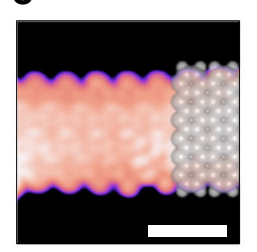

f

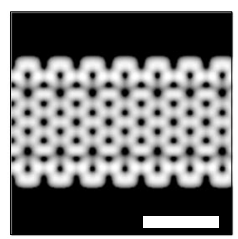

Fig. 3 STM and DFT simulated images of 17- and 13-AGNRs. a Overview STM image $\left(V_{s}=-1.2 \mathrm{~V}, I_{t}=10 \mathrm{pA}\right)$ of 17-AGNRs on Au(111). b High-resolution STM image $\left(V_{s}=-1.4 \mathrm{~V}, I_{\mathrm{t}}=1.0 \mathrm{nA}\right)$ of single 17-AGNR together with structural model. c DFT-simulated image $(U=-1.4 \mathrm{eV})$ of the 17-AGNR. d Overview STM image $\left(V_{s}=-1.5 V, I_{t}=20 \mathrm{pA}\right)$ of 13-AGNRs on Au(111). e High-resolution STM image $\left(V_{s}=-1.6 \mathrm{~V}, I_{t}=1.2 \mathrm{nA}\right)$ of single 13-AGNR together with structural model and $\mathbf{f}$ corresponding DFT-simulated image $(U=-1.6 \mathrm{eV})$. Overview and high-resolution images were taken at 77 and $5 \mathrm{~K}$, respectively. Scale bar, $10 \mathrm{~nm}$ in $\mathbf{a}, \mathbf{d}, 1 \mathrm{~nm}$ in $\mathbf{b}, \mathbf{c}, \mathbf{e}, \mathbf{f}$.

when preparing samples under ex-situ conditions. In addition to the STM and nc-AFM characterizations, the quality of the 17and 13-AGNRs on a larger scale were investigated by X-ray photoelectron (Supplementary Note 2, Fig. 12) and Raman (Supplementary Note 3, Fig. 13) spectroscopies.
Electronic structures of 17- and 13-AGNRs. To acquire information about the electronic structures of the 17- and 13-AGNRs, we carried out STS measurements. In the Tersoff-Hamann approximation $^{30}$, a differential conductance $(\mathrm{d} I / \mathrm{d} V)$ obtained by STS is proportional to the local density of states (LDOSs) at the 


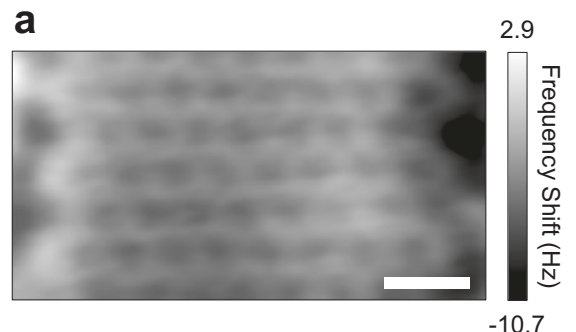

b
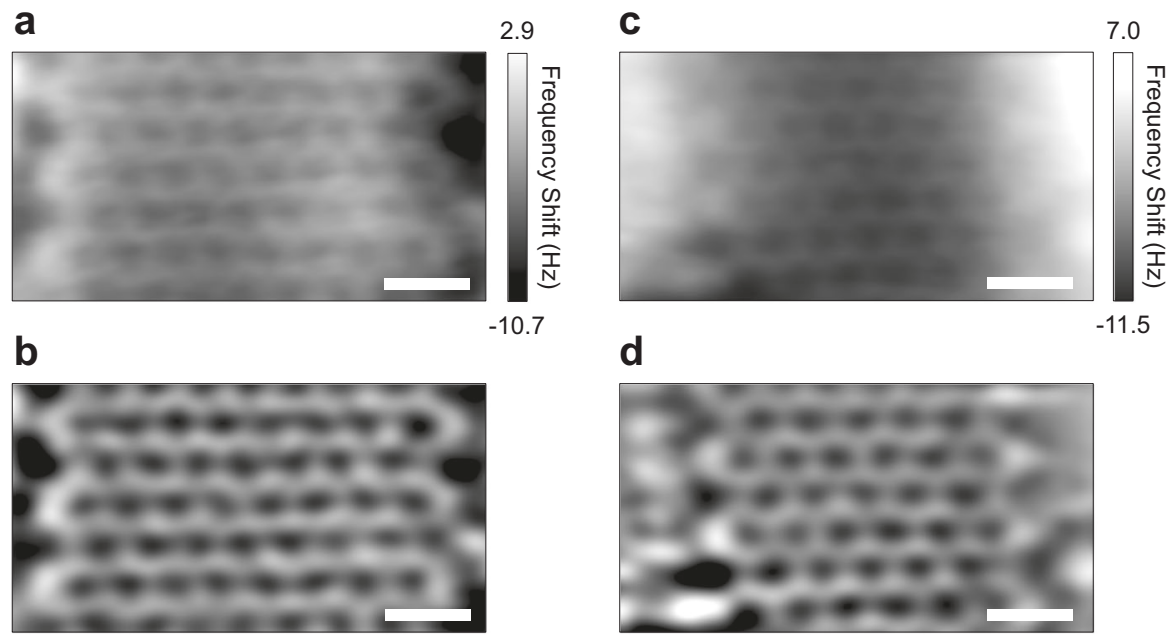

d

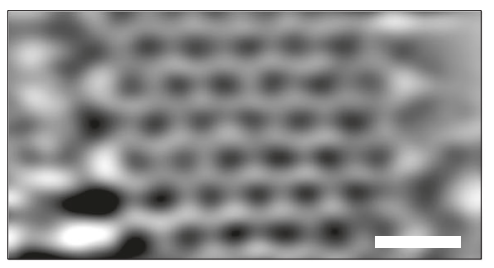

Fig. 4 nc-AFM characterization of 17- and 13-AGNRs. a Constant-height frequency shift image of single 17-AGNR measured by nc-AFM with a COfunctionalized tip. $\mathbf{b}$ Laplace-filtered image of $\mathbf{a}$. $\mathbf{c}$ Analogous frequency shift image of single 13-AGNR and $\mathbf{d}$ Laplace-filtered image of $\mathbf{c}$. Frequency shift images were taken at $V_{\mathrm{s}}=0 \mathrm{~V}$, oscillation amplitude of $0.10 \mathrm{~nm}$ and $5 \mathrm{~K}$. All scale bars, $0.5 \mathrm{~nm}$.

a

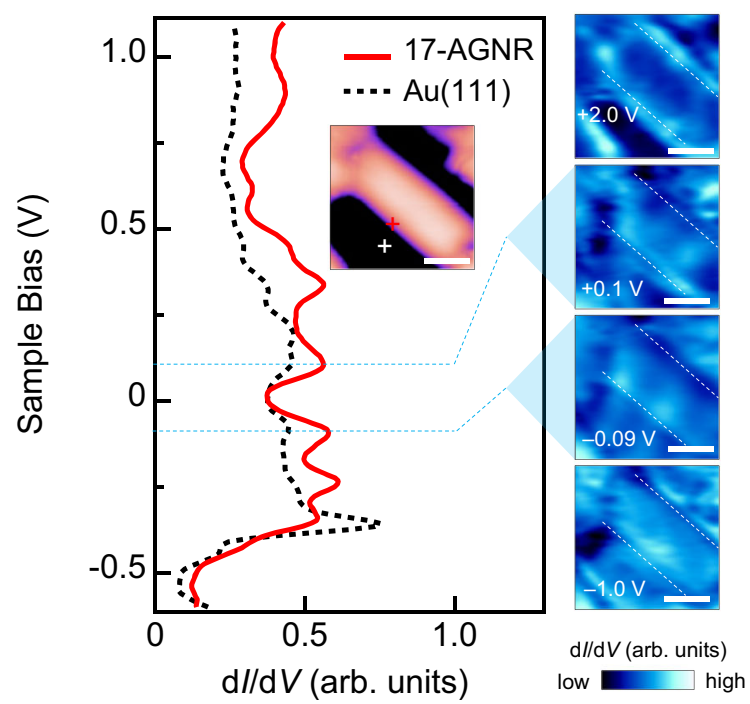

C

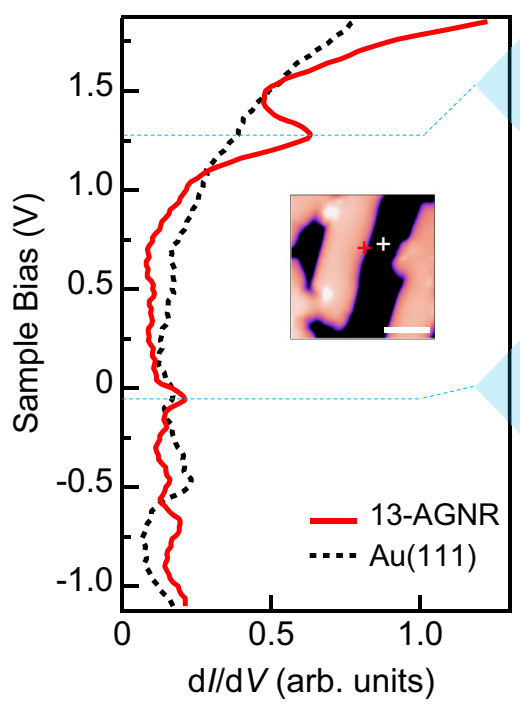

d

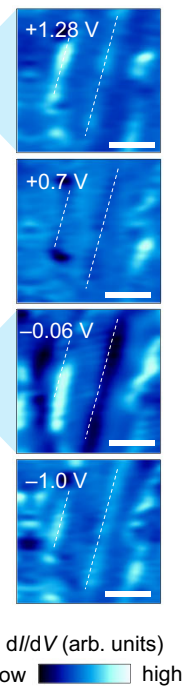

Fig. 5 Electronic state characterization of 17- and 13-AGNRs. a Differential conductance ( $d / / d V$ ) point spectra recorded on edge of single 17-AGNR (red line) and $\mathrm{Au}(111)$ surface (black dotted line). Crosses in inset of STM image indicate tip positions for STS. Open-feedback setpoints were $V_{\mathrm{s}}=-1.1 \mathrm{~V}, I_{\mathrm{t}}=$ $0.41 \mathrm{nA}$. b Constant-height $\mathrm{d} l / \mathrm{d} V$ maps $\left(I_{\mathrm{t}}=0.41 \mathrm{nA}\right)$ of the 17-AGNR obtained at energies indicated in each map. Dashed lines indicate outer edges of the AGNR. $\mathbf{c} \mathrm{d} / \mathrm{d} V$ spectra of single 13-AGNR on $\mathrm{Au}(111)$ recorded at tip positions marked with crosses in inset STM image (setpoints: $V_{\mathrm{s}}=2.2 \mathrm{~V}, l_{\mathrm{t}}=0.8 \mathrm{nA}$ ). d $\mathrm{d} l / \mathrm{d} V$ maps $\left(I_{\mathrm{t}}=0.8 \mathrm{nA}\right)$ of the 13-AGNR obtained at respective energies shown in each map. All STS data were obtained at $5 \mathrm{~K}$. All scale bars, $2 \mathrm{~nm}$.

position of the STM tip. The LDOSs in the 17- and 13-AGNRs were characterized using the $\mathrm{d} I / \mathrm{d} V$ point spectra, as shown in Fig. 5a, c. All spectra were obtained after calibrating the STM tip by confirming the appearance of the so-called Shockley surface states on $\mathrm{Au}(111)$ around $V_{\mathrm{s}} \sim-0.4 \mathrm{~V}$ (ref. ${ }^{31}$ ).

By comparing the $\mathrm{d} I / \mathrm{d} V$ spectrum obtained on the edge of the 17-AGNR (red line) to that obtained on the bare $\mathrm{Au}(111)$ surface (black dotted line) in Fig. $5 \mathrm{a}$, we notice the ribbon-related peaks centered at $-0.09 \pm 0.02 \mathrm{~V}$ (occupied states) and $0.10 \pm 0.02 \mathrm{~V}$ (unoccupied states), which can be regarded as the valence band maximum and conduction band minimum, respectively, since they bracket the Fermi level $E_{\mathrm{F}}\left(\right.$ at $V_{\mathrm{s}}=0 \mathrm{~V}$ ). From the energy difference between these peaks, the experimental energy gap is estimated to be $\Delta_{\mathrm{STS}}=0.19 \pm 0.03 \mathrm{eV}$ for the 17-AGNR on $\mathrm{Au}$
(111). In the $G W$ calculations, the quasiparticle gap of a freestanding 17-AGNR is predicted to be $\Delta_{G W}=0.63 \mathrm{eV}$ (ref. ${ }^{24}$ ). In general, the values of $\Delta_{\text {STS }}$ determined by STS in GNRs on metal surfaces are significantly underestimated to those of $\Delta_{G W}$ for freestanding GNRs. The reduction in $\Delta_{\mathrm{STS}}$ to $\Delta_{G W}$ is caused by a substrate-induced weakening of the electrostatic potential due to long-range screening effects ${ }^{22}$. Therefore, the $\Delta_{G W}$ values should be corrected by considering the substrate screening when compared with those of $\Delta_{\mathrm{STS}}$. According to ref. ${ }^{32}$, the quasiparticle gaps of substrate-supported GNRs have been corrected using an advanced image-charge model that includes the substrate screening as well as the internal screening of the GNRs. The renormalized quasiparticle gap of 17-AGNRs is predicted to be $\Delta_{G W^{\prime}}{ }^{\prime}=0.20 \mathrm{eV}$ (ref. ${ }^{22}$ ), which is in good 
agreement with our experimental energy gap. In fact, the renormalized gap was computed with a model of 17-AGNRs adsorbed on $\mathrm{a} \mathrm{Au}_{3} \mathrm{Si}$ monolayer intercalated between the ribbon and $\mathrm{Au}(111)$ substrate $^{22}$. Intercalating a semiconducting or insulating layer into the GNR-substrate interface increases the relative distance between the GNR and image-plane of the substrate. The increase in the distance leads to the reduction in the image-charge corrections, expanding the renormalized gap. However, in wide GNRs, such as 17-AGNRs, the internal screening of GNRs is, owing to their large polarizabilities, more dominant than the external screening from the substrate ${ }^{32}$. In such cases, the change in the renormalized gap depending on the presence or absence of the intercalated layer is almost negligible. Therefore, we can safely conclude that the agreement of our experimental energy gap with the theoretical renormalized gap is consistent.

The spatial distributions of the electronic structure of the 17AGNR were experimentally explored by $\mathrm{d} I / \mathrm{d} V$ mapping at different sample biases (Fig. 5b). The $\mathrm{d} I / \mathrm{d} V$ maps obtained at $V_{\mathrm{s}}=2.0$ and $-1.0 \mathrm{~V}$ exhibit significant LDOSs along the two edges of the ribbon. On the other hand, in the $\mathrm{d} I / \mathrm{d} V$ maps at $V_{\mathrm{s}}=$ 0.1 and $-0.09 \mathrm{~V}$ corresponding to the energies of the conduction band minimum and valence band maximum, the enhancement of the $\mathrm{d} I / \mathrm{d} V$ intensity along the edges is partially suppressed because of the oscillatory contrast derived from the quantum interference of the $\mathrm{Au}(111)$ surface states at these biases ${ }^{33}$.

Figure $5 c$ illustrates the $\mathrm{d} I / \mathrm{d} V$ spectra taken on the edge of a single 13-AGNR and Au(111) surface. The spectrum of the 13AGNR shows two prominent peaks centered at $-0.06 \pm 0.02$ and $1.28 \pm 0.02 \mathrm{~V}$. These peak positions are similar to the energy positions of the valence band maximum and conduction band minimum previously measured by STS for 13-AGNRs on $\mathrm{Au}$ $(111)^{20}$. The energy gap is estimated to be $\Delta_{\mathrm{STS}}=1.34 \pm 0.03 \mathrm{eV}$ from the energy difference between those peaks. This energy gap is also consistent with the previous one $(1.4 \pm 0.1 \mathrm{eV})^{20}$. With the $G W$ calculations, the quasiparticle gap is predicted to be $\Delta_{G W}=$ $2.25 \mathrm{eV}$ for a freestanding 13-AGNR ${ }^{24}$, and the renormalized gap of the 13-AGNR supported by the $\mathrm{Au}(111)$ substrate is corrected as $\Delta_{G W^{\prime}}=1.29 \mathrm{eV}$ using the advanced image-charge model ${ }^{32}$. The theoretical gap shows good agreement with our experimental one.

For this 13-AGNR, the $\mathrm{d} I / \mathrm{d} V$ maps measured at the characteristic sample biases are depicted in Fig. $5 \mathrm{~d}$. The $\mathrm{d} I / \mathrm{d} V$ maps clearly show significant LDOSs along the edges at the conduction band minimum and valence band maximum, as well as in the occupied state (at $V_{\mathrm{s}}=-1.0 \mathrm{~V}$ ). In contrast, there is no prominent $\mathrm{d} I / \mathrm{d} V$ intensity on the ribbon in the map at $V_{\mathrm{s}}=0.7 \mathrm{~V}$ since this sample bias corresponds to the energy within the bandgap. These LDOS behaviors in the 13-AGNR by varying the sample bias were also observed in previous STS measurements ${ }^{20}$.

Comparison between experimental and theoretical band structures. For more detailed discussions on the dispersion of the electronic states of the 17- and 13-AGNRs, we carried out Fourier-transformed STS (FT-STS) measurements. Figure 6a shows a series of $\mathrm{d} I / \mathrm{d} V$ spectra measured along one armchair edge of a single 17 -AGNR at intervals $\delta x=0.11 \mathrm{~nm}$ (see Supplementary Fig. 14a for the measured 17-AGNR). In the LDOS [i.e., $\mathrm{d} I / \mathrm{d} V(V, x)]$ map, while the standing wave patterns derived from scattering at the termini of the ribbon are clear in the unoccupied states $\left(V_{\mathrm{s}}>1.3 \mathrm{~V}\right)$ and occupied states $\left(V_{\mathrm{s}}<-0.5 \mathrm{~V}\right)$, the weak patterns are also clear in the vicinity of $E_{\mathrm{F}}$. Moreover, the absence of the LDOSs within the bandgap can be confirmed throughout the measuring positions. The LDOS maps of a single 13-AGNR in the unoccupied and occupied states are presented in Fig. 6c, d (the measured 13-AGNR is shown in Supplementary
Fig. $14 \mathrm{~b}, \mathrm{c})$. Note that the LDOSs of the 13-AGNR are absent in the region from $V_{\mathrm{s}} \sim 1 \mathrm{~V}$ to $E_{\mathrm{F}}$, reflecting the bandgap.

To obtain the electronic band dispersion, we carried out a discrete Fourier transform of $\mathrm{d} I / \mathrm{d} V(V, x)$ in real space to reciprocal space (see detailed procedures in ref. ${ }^{34}$ ). Figure $6 \mathrm{~b}$ presents the FT-LDOS map of Fig. 6a for the 17-AGNR in the range of the wave vector $0 \leq k(=q / 2) \leq \pi / a$ corresponding to the first Brillouin zone of the ribbon. The FT-LDOS maps of the 13AGNR in the unoccupied and occupied states are shown in Fig. 6e, f. In both 17- and 13-AGNRs, the FT-LDOS maps reveal the appearance of dispersing bands and the bandgaps. It is, however, difficult to discuss the respective bands by resolving each one due to insufficient signal-to-noise ratio. This experimental problem could be due to the finite tip size and a deviation from a constant tip-sample distance $(\sim 0.1 \mathrm{~nm})^{19,34}$. We thus computed the quasiparticle bands of freestanding 17- and 13AGNRs using the $G W$ calculations to compare them with the experimental band dispersions. Figure $6 \mathrm{~g}$, $\mathrm{h}$ shows the calculated band structures of the 17- and 13-AGNRs. From our calculations, the quasiparticle gap of these AGNRs is predicted to be $\Delta_{G W}=$ 0.66 and $2.01 \mathrm{eV}$, which is in acceptable agreement with the previous calculation results ${ }^{24}$.

As mentioned above, the $G W$ calculations overestimate the bandgap of GNRs compared with the STS experiments. We thus attempted to rigidly shift the $G W$ quasiparticle bands to compare them with the experimental band dispersions (see red dotted curves in Fig. 6b, e, f). All quasiparticle conduction and valence bands are shifted to match the onset of the quasiparticle conduction and valence bands with the energies of the experimental conduction band minimum and valence band maximum (Fig. 5a, c). Surprisingly, the rigid-shifted quasiparticle bands reasonably reproduce the experimentally observed band structures in both 17- and 13-AGNRs, although each quasiparticle band is not corrected for image-charge effects.

It is difficult to accurately estimate the effective masses by using these experimental data. Thus, as an indirect comparison, we carried out a parabolic least-squares fit $\left[E(k)=E(0)+\hbar^{2} k^{2} / 2 m^{*}\right]$ to the $G W$ quasiparticle conduction and valence bands of these 17and 13-AGNRs (depicted with blue dotted curves in Fig. 6g, h). The respective effective masses $\left(m^{*}{ }_{\mathrm{CB}}\right.$ and $m_{\mathrm{VB}}^{*}$ for the conduction and valence bands) are estimated as $m_{\mathrm{CB}}^{*}=m_{\mathrm{VB}}^{*}=$ $0.06 m_{\mathrm{e}}$ in the 17-AGNR and $m_{\mathrm{CB}}^{*}=0.14 m_{\mathrm{e}}$ and $m_{\mathrm{VB}}^{*}=0.13 m_{\mathrm{e}}$ in the 13-AGNR (where $m_{\mathrm{e}}$ is the free electron mass). Given the reasonably good agreement between the band structures obtained experimentally and theoretically, the actual effective mass is expected to be close to the $G W$ values. Notably, the small effective masses of both electrons and holes $\left(m^{*} \sim 0.06 m_{\mathrm{e}}\right)$, as well as the small bandgap $\left(\Delta_{\mathrm{STS}}=0.19 \mathrm{eV}\right)$, were obtained in the 17 -AGNR on $\mathrm{Au}(111)$. These values are substantially smaller than $m^{*} \sim 0.4 m_{\mathrm{e}}$ and $\Delta_{\mathrm{STS}}=2.4 \mathrm{eV}$ for $7-\mathrm{AGNRs}^{34}$ and $m^{*} \sim 0.1 m_{\mathrm{e}}$ and $\Delta_{\mathrm{STS}}=1.4$ $\mathrm{eV}$ for 9 -AGNRs ${ }^{19}$, which have been previously exploited in prototypes of GNR-based FETs. The electron/hole effective mass in the 17-AGNR is even smaller than those in GaAs and InP (e.g., ref. ${ }^{35}$ ). Our results indicate that excellent devices including transistors can be obtained using 17-AGNRs in the near future.

In conclusion, we have discussed the synthesis of AGNRs of $N=17$ and 13 widths on $\mathrm{Au}(111)$ by using the bottom-up technique starting from two types of dibromobenzene-based precursor monomers. STM and nc-AFM observations revealed their widths and armchair-edged structures at atomic precision. The local electronic structures characterized by STS allow for the determination of their bandgaps. The experimentally determined gaps of the 17- and 13-AGNRs on $\mathrm{Au}(111)$ are in fair agreement with the $G W$ quasiparticle gaps corrected for substrate screening. Furthermore, FT-STS provides the electronic band dispersions, 
a

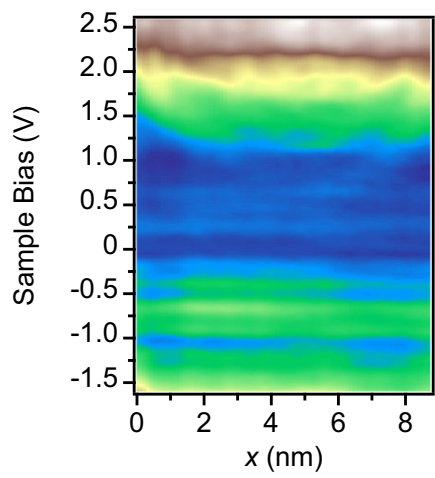

C

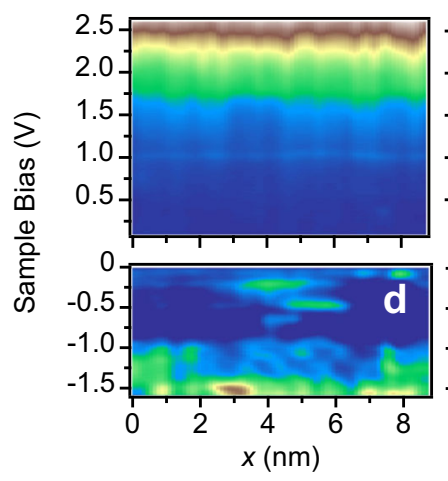

b

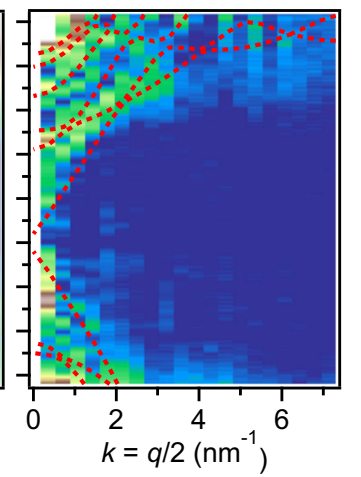

e

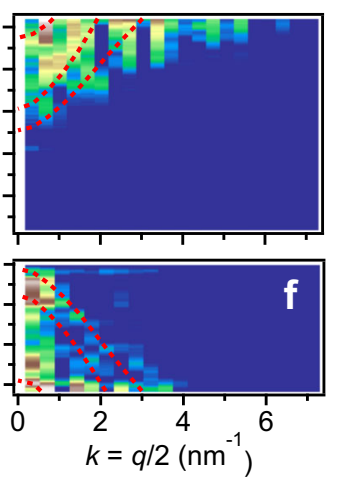

g

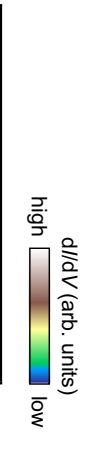

h
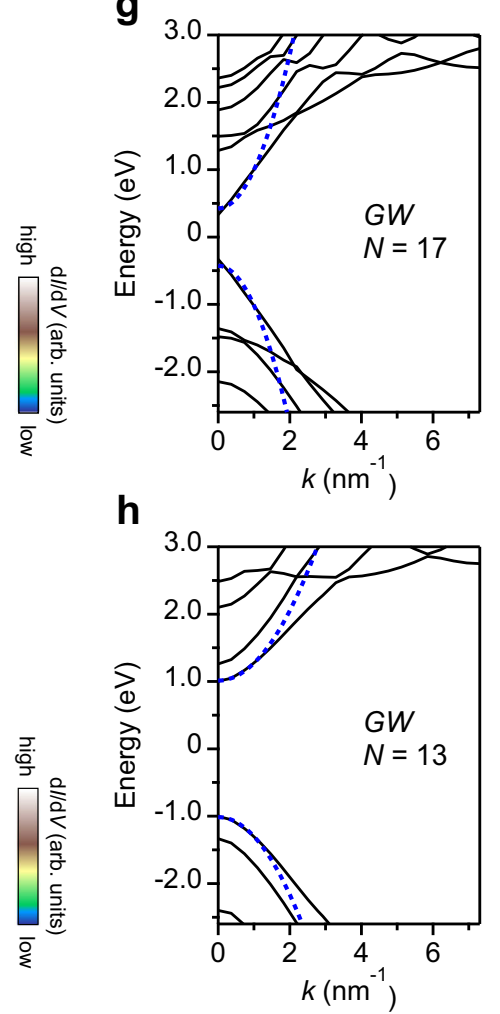

Fig. 6 Electronic band structures of 17- and 13-AGNRs. a Series of $d / / d V$ spectra obtained along one armchair edge of single 17-AGNR with length of 20a on $\mathrm{Au}(111)$ (setpoints: $V_{\mathrm{s}}=2.6 \mathrm{~V}, I_{\mathrm{t}}=0.7 \mathrm{nA}$, spacing $\left.\delta x=0.11\right)$. b Fourier-transformed map of a for $0 \leq k(=q / 2) \leq \pi / a$. c, d Analogous dl/dV spectral maps of single 13-AGNR with length of $20 a$ on $A u(111)$ for $\mathbf{c}$ unoccupied state (setpoints: $V_{s}=2.6 \mathrm{~V}, I_{t}=0.7 \mathrm{nA}$, spacing $\delta x=0.11$ ) and $\mathbf{d}$ occupied state (setpoints: $V_{\mathrm{s}}=-1.6 \mathrm{~V}, I_{\mathrm{t}}=0.2 \mathrm{nA}$, spacing $\delta x=0.11$ ). e, $\mathbf{f}$ Fourier-transformed maps of $\mathbf{c}, \mathbf{d}$. $\mathbf{g}, \mathbf{h} G W$ quasiparticle band structures (black curves) of freestanding $\mathbf{g}$ 17- and $\mathbf{h} 13-A G N R s$, aligned at center of gap ( $0 \mathrm{eV}$ ). Blue dotted curves show parabolic fits in rage of $0 \leq k \leq 1 \mathrm{~nm}^{-1}$ to obtain band effective masses. In Fourier-transformed maps of $\mathbf{b}$ and $\mathbf{e}$, $\mathbf{f}$, red dotted curves indicate rigid-shifted quasiparticle bands of $\mathbf{g}$ and $\mathbf{h}$ to compare them with experimental band dispersions. All STS data were obtained at $5 \mathrm{~K}$.

which reasonably match the simply rigid-shifted $G W$ quasiparticle bands in both types of AGNRs. We further found that the 17-AGNRs on $\mathrm{Au}(111)$ have a small bandgap of $0.19 \mathrm{eV}$ and small effective masses of $\sim 0.06 \mathrm{~m}_{\mathrm{e}}$ for electrons and holes. We expect that the 17-AGNRs successfully synthesized in this study will pave the way for the development of GNR-based electronic devices.

\section{Methods \\ Sample preparation. Experiments were conducted under ultra-high vacuum conditions (base pressure $<5 \times 10^{-8} \mathrm{~Pa}$ ) with a low-temperature STM from Scienta Omicron. A Au(111) single crystal (MaTeck, Germany) and Au(111) epitaxial films on mica (Phasis, Switzerland) were used as substrates for the synthesis of the 17- and 13-AGNRs (we obtained similar results regardless of the types of substrates). Atomically clean $\mathrm{Au}(111)$ surfaces were prepared by repeated cycles of Ar ion sputtering and annealing at $500^{\circ} \mathrm{C}$. BADBB and BNDBB monomers (for details on the synthesis and characterization, see Supplementary Note 1) were thermally evaporated onto the clean $\mathrm{Au}(111)$ surface held at room temperature from a quartz crucible heated to $180^{\circ} \mathrm{C}$ for BADBB and $120^{\circ} \mathrm{C}$ for BNDBB, resulting in a deposition rate of $\sim 1 \AA \mathrm{min}^{-1}$ in both monomers. The coverage of both monomers was controlled to be sub-monolayer, as determined from STM (Supplementary Fig. 11a, b). After deposition, the surface temperature was step-wisely ramped $\left(<5^{\circ} \mathrm{C} \mathrm{min}{ }^{-1}\right)$ from room temperature to $400^{\circ} \mathrm{C}$ in five steps to $200,250,300,350$, and $400{ }^{\circ} \mathrm{C}$. Each temperature step was held for $30 \mathrm{~min}$ at or $<350^{\circ} \mathrm{C}$ and for $2 \mathrm{~h}$ at $400^{\circ} \mathrm{C}$. Long and low-defective AGNRs were obtained owing to this stepwise annealing.}

Imaging and spectroscopy. STM measurements were conducted in the constantcurrent mode under $3 \times 10^{-9} \mathrm{~Pa}$ at a sample temperature of 77 or $5 \mathrm{~K}$. The temperature and scanning parameters are described in each figure caption. An electrochemically etched W tip was used for topographic and spectroscopic measurements. For STS measurements, all $\mathrm{d} I / \mathrm{d} V$ signals were recorded at $5 \mathrm{~K}$ using a lock-in amplifier with a sinusoidal voltage of $10 \mathrm{mV}$ (r.m.s.) and frequency of $463 \mathrm{~Hz}$. The $\mathrm{d} I / \mathrm{d} V$ spectra and maps were acquired under the open-feedback and constant-current conditions, respectively.

The nc-AFM experiments were conducted in another ultra-high vacuum chamber under $4 \times 10^{-9} \mathrm{~Pa}$ at a sample temperature of $5 \mathrm{~K}$. Samples [17- and 13-AGNRs grown on $\mathrm{Au}(111) /$ mica substrates] mounted in the chamber were degassed at $200{ }^{\circ} \mathrm{C}$ for $4 \mathrm{~h}$, then annealed at $400{ }^{\circ} \mathrm{C}$ for $20 \mathrm{~min}$ for the 17 -AGNRs and at $420^{\circ} \mathrm{C}$ for $30 \mathrm{~min}$ for the 13-AGNRs to remove impurities on the surface. For frequency modulation AFM, a tuning fork with an etched $\mathrm{W}$ tip was used as a force sensor (resonance frequency of $21.3 \mathrm{kHz}$, spring constant of $1800 \mathrm{~N} \mathrm{~m}^{-1}$, quality factor of $\left.1-5 \times 10^{4}\right)$. To obtain high-resolution AFM images, a CO molecule coadsorbed on the surfaces at $6 \mathrm{~K}$ was picked up to attach to the tip apex ${ }^{36}$. Frequency shift was measured in the constant-height mode. For the AFM image of the 17-AGNRs (13-AGNRs), the tip height was $0.07 \mathrm{~nm}$ higher $(0.03 \mathrm{~nm}$ lower) than the setpoint determined from STM at $V_{\mathrm{s}}=30 \mathrm{mV}$ and $I_{\mathrm{t}}=20 \mathrm{pA}$ over the bare Au surface. All scanning images were edited using SPIP software (Image Metrology, Denmark).

Theoretical calculations. Geometry optimizations for the 17- and 13-AGNRs were carried out using DFT within the generalized gradient approximation using the Perdew-Burke-Ernzerhof functional ${ }^{37}$ for the exchange-correlation function as implemented in the OpenMX package (http://www.openmx-square.org/). The electron-ion interaction is described by norm-conserving pseudopotentials ${ }^{38}$ under partial core correction ${ }^{39}$. Pseudo-atomic orbitals (PAOs) centered on atomic sites are used as the basis function set ${ }^{40}$. The PAO basis functions are specified by C6.0-s $2 p 2 d 1$ and H5.0-s2p1. For example, C6.0-s $2 p 2 d 1$ indicates the PAO of the carbon atom with the cutoff radii of $6.0 \mathrm{Bohr}$ and with two $s$, two $p$, and one $d$ component. The van der Waals corrections were included with a semiempirical DFT-D2 method $^{41}$. Simulated STM images were obtained by the Tersoff-Hamann theory ${ }^{30}$. Partial charge density was calculated in an energy window $U$ measured from the chemical potential (shown in each figure caption) and visualized using WSxM software ${ }^{42}$. The lattice constant of the AGNRs was $4.30 \AA$, and the 
geometries were optimized under a three-dimensional periodic boundary condition with a criterion of $5.14 \times 10^{-3} \mathrm{eV} \AA^{-1}$ for forces on atoms.

Quasiparticle band structure calculations were computed in the $G W$ approximation using the Berkeley $G W$ package ${ }^{23,43}$. The electronic structure from DFT was recalculated using 60 Ry plane-wave cutoff and $64 k$-points in the first Brillouin zone. The quasiparticle energies are determined by considering the lowest 129 and 45 unoccupied conduction bands for the 17- and 13-AGNRs, respectively. The static dielectric matrix $\varepsilon$ was calculated in the random phase approximation with $8 \mathrm{Ry}$ cutoff for the plane-wave basis. The dynamical electronic screening is captured using the general plasmon pole model ${ }^{23}$.

\section{Data availability}

All data generated or analyzed during this study are included in the published article and the Supplementary Information, and are available from the corresponding authors upon reasonable request.

Received: 31 January 2020; Accepted: 19 May 2020;

Published online: 23 June 2020

\section{References}

1. Nakada, K., Fujita, M., Dresselhaus, G. \& Dresselhaus, M. S. Edge state in graphene ribbons: nanometer size effect and edge shape dependence. Phys. Rev. B 54, 17954-17961 (1996).

2. Son, Y.-W., Cohen, M. L. \& Louie, S. G. Half-metallic graphene nanoribbons. Nature 444, 347-349 (2006).

3. Barone, V., Hod, O. \& Scuseria, G. E. Electronic structure and stability of semiconducting graphene nanoribbons. Nano Lett. 6, 2748-2754 (2006).

4. Tao, C. et al. Spatially resolving edge states of chiral graphene nanoribbons Nat. Phys. 7, 616-620 (2011).

5. Ezawa, M. Peculiar width dependence of the electronic properties of carbon nanoribbons. Phys. Rev. B 73, 045432 (2006).

6. Son, Y.-W., Cohen, M. L. \& Louie, S. G. Energy gaps in graphene nanoribbons. Phys. Rev. Lett. 97, 216803 (2006).

7. Yang, L., Park, C.-H., Son, Y.-W., Cohen, M. L. \& Louie, S. G. Quasiparticle energies and band gaps in graphene nanoribbons. Phys. Rev. Lett. 99, 186801 (2007).

8. Wassmann, T., Seitsonen, A. P., Saitta, A. M., Lazzeri, M. \& Mauri, F. Clar's theory, $\pi$-electron distribution, and geometry of graphene nanoribbons. J. Am. Chem. Soc. 132, 3440-3451 (2010).

9. Han, M. Y., Özyilmaz, B., Zhang, Y. \& Kim, P. Energy band-gap engineering of graphene nanoribbons. Phys. Rev. Lett. 98, 206805 (2007).

10. Li, X., Wang, X., Zhang, L., Lee, S. \& Dai, H. Chemically derived, ultrasmooth graphene nanoribbon semiconductors. Science 319, 1229 (2008).

11. Kosynkin, D. V. et al. Longitudinal unzipping of carbon nanotubes to form graphene nanoribbons. Nature 458, 872-876 (2009).

12. Cai, J. et al. Atomically precise bottom-up fabrication of graphene nanoribbons. Nature 466, 470 (2010).

13. Ruffieux, P. et al. Electronic structure of atomically precise graphene nanoribbons. ACS Nano 6, 6930-6935 (2012).

14. Linden, S. et al. Electronic structure of spatially aligned graphene nanoribbons on $\mathrm{Au}(788)$. Phys. Rev. Lett. 108, 216801 (2012).

15. Ruffieux, P. et al. On-surface synthesis of graphene nanoribbons with zigzag edge topology. Nature 531, 489 (2016).

16. Vasseur, G. et al. Quasi one-dimensional band dispersion and surface metallization in long-range ordered polymeric wires. Nat. Commun. 7, 10235 (2016).

17. Vasseur, G. et al. $\Pi$ band dispersion along conjugated organic nanowires synthesized on a metal oxide semiconductor. J. Am. Chem. Soc. 138, 5685-5692 (2016)

18. Zhang, H. et al. On-surface synthesis of Rylene-type graphene nanoribbons. J. Am. Chem. Soc. 137, 4022-4025 (2015).

19. Talirz, L. et al. On-surface synthesis and characterization of 9-atom wide armchair graphene nanoribbons. ACS Nano 11, 1380-1388 (2017).

20. Chen, Y.-C. et al. Tuning the band gap of graphene nanoribbons synthesized from molecular precursors. ACS Nano 7, 6123-6128 (2013).

21. Huang, H. et al. Spatially resolved electronic structures of atomically precise armchair graphene nanoribbons. Sci. Rep. 2, 983 (2012).

22. Deniz, O. et al. Revealing the electronic structure of silicon intercalated armchair graphene nanoribbons by scanning tunneling spectroscopy. Nano Lett. 17, 2197-2203 (2017).

23. Hybertsen, M. S. \& Louie, S. G. Electron correlation in semiconductors and insulators: band gaps and quasiparticle energies. Phys. Rev. B 34, 5390-5413 (1986).
24. Zhu, X. \& Su, H. Scaling of excitons in graphene nanoribbons with armchair shaped edges. J. Phys. Chem. A 115, 11998-12003 (2011).

25. Bennett, P. B. et al. Bottom-up graphene nanoribbon field-effect transistors. Appl. Phys. Lett. 103, 253114 (2013).

26. Llinas, J. P. et al. Short-channel field-effect transistors with 9-atom and 13atom wide graphene nanoribbons. Nat. Commun. 8, 633 (2017).

27. Javey, A., Guo, J., Wang, Q., Lundstrom, M. \& Dai, H. Ballistic carbon nanotube field-effect transistors. Nature 424, 654-657 (2003).

28. Dürkop, T., Getty, S. A., Cobas, E. \& Fuhrer, M. S. Extraordinary mobility in semiconducting carbon nanotubes. Nano Lett. 4, 35-39 (2004).

29. Di Giovannantonio, M. et al. On-surface growth dynamics of graphene nanoribbons: the role of halogen functionalization. ACS Nano 12, 74-81 (2018).

30. Tersoff, J. \& Hamann, D. R. Theory of the scanning tunneling microscope. Phys. Rev. B 31, 805-813 (1985).

31. Chen, W., Madhavan, V., Jamneala, T. \& Crommie, M. F. Scanning tunneling microscopy observation of an electronic superlattice at the surface of clean gold. Phys. Rev. Lett. 80, 1469-1472 (1998).

32. Kharche, N. \& Meunier, V. Width and crystal orientation dependent band gap renormalization in substrate-supported graphene nanoribbons. J. Phys. Chem. Lett. 7, 1526-1533 (2016).

33. Crommie, M. F., Lutz, C. P. \& Eigler, D. M. Imaging standing waves in a twodimensional electron gas. Nature 363, 524-527 (1993).

34. Söde, $\mathrm{H}$. et al. Electronic band dispersion of graphene nanoribbons via Fourier-transformed scanning tunneling spectroscopy. Phys. Rev. B 91, 045429 (2015)

35. Sze, S. M. \& Ng, K. K. Physics of Semiconductor Devices 3rd edn (John Wiley \& Sons, Inc., Hoboken, NJ, 2007)

36. Gross, L., Mohn, F., Moll, N., Liljeroth, P. \& Meyer, G. The chemical structure of a molecule resolved by atomic force microscopy. Science 325, 1110 (2009).

37. Perdew, J. P., Burke, K. \& Ernzerhof, M. Generalized gradient approximation made simple. Phys. Rev. Lett. 77, 3865-3868 (1996).

38. Morrison, I., Bylander, D. M. \& Kleinman, L. Nonlocal Hermitian normconserving Vanderbilt pseudopotential. Phys. Rev. B 47, 6728-6731 (1993).

39. Louie, S. G., Froyen, S. \& Cohen, M. L. Nonlinear ionic pseudopotentials in spin-density-functional calculations. Phys. Rev. B 26, 1738-1742 (1982).

40. Ozaki, T. Variationally optimized atomic orbitals for large-scale electronic structures. Phys. Rev. B 67, 155108 (2003).

41. Grimme, S. Semiempirical GGA-type density functional constructed with a long-range dispersion correction. J. Comput. Chem. 27, 1787-1799 (2006).

42. Horcas, I. et al. WSXM: a software for scanning probe microscopy and a tool for nanotechnology. Rev. Sci. Instrum. 78, 013705 (2007).

43. Deslippe, J. et al. BerkeleyGW: a massively parallel computer package for the calculation of the quasiparticle and optical properties of materials and nanostructures. Comput. Phys. Commun. 183, 1269-1289 (2012).

\section{Acknowledgements}

This work was supported by CREST JST (no. JPMJCR15F1) and MEXT/JSPS KAKENHI grant nos. 26105004, 16H02286, 18K14190, and 18H01807. We thank Dr. Atsushi Ando of the National Advanced Industrial Science and Technology for supporting the Raman experiments. We also thank Prof. Riichiro Saito of Tohoku University for helpful advice on theoretical interpretations of the Raman spectra.

\section{Author contributions}

J.Y. synthesized the AGNRs, conducted the STM/STS measurements and carried out the data analysis. A.S. and Y.S. conducted the nc-AFM imaging. H.H., M.S., and N.H. synthesized the precursor monomers. N.A. conducted the single-crystal X-ray analysis. J.Y., H.J. and M. Ohtomo conducted the theoretical simulations. S.S., H.Y., and M. Ohfuchi supervised the project. J.Y., H.H., H.J., and A.S. wrote the manuscript, and all authors discussed the results and commented on the manuscript.

\section{Competing interests}

The authors declare no competing interests.

\section{Additional information}

Supplementary information is available for this paper at https://doi.org/10.1038/s43246020-0039-9.

Correspondence and requests for materials should be addressed to J.Y. or H.Y.

Reprints and permission information is available at http://www.nature.com/reprints

Publisher's note Springer Nature remains neutral with regard to jurisdictional claims in published maps and institutional affiliations. 
(c) (i) Open Access This article is licensed under a Creative Commons Attribution 4.0 International License, which permits use, sharing, adaptation, distribution and reproduction in any medium or format, as long as you give appropriate credit to the original author(s) and the source, provide a link to the Creative Commons license, and indicate if changes were made. The images or other third party material in this article are included in the article's Creative Commons license, unless indicated otherwise in a credit line to the material. If material is not included in the article's Creative Commons license and your intended use is not permitted by statutory regulation or exceeds the permitted use, you will need to obtain permission directly from the copyright holder. To view a copy of this license, visit http://creativecommons.org/ licenses/by/4.0/.

(C) The Author(s) 2020 